\title{
Raman Signature of Defected Twisted Bilayer Graphene
}

Scott W. Schmucker ${ }^{\mathrm{a},}{ }^{*}$, Cory D. Cress ${ }^{\mathrm{a}}$, James C. Culbertson ${ }^{\mathrm{a}}$, Jeffrey W. Beeman ${ }^{\mathrm{b}}$, Oscar D. Dubon $^{\mathrm{b}, \mathrm{c}}$, Jeremy T. Robinson ${ }^{\mathrm{a}}$

${ }^{a}$ US Naval Research Laboratory, Washington, DC 20375, USA

${ }^{\mathrm{b}}$ Lawrence Berkeley National Laboratory, Berkeley, CA, USA

${ }^{c}$ University of California-Berkeley, Berkeley, CA, USA

\begin{abstract}
Layered two-dimensional crystal systems can exhibit complex interlayer interactions, which are influenced by local crystal structure and/or electronic variations. Here, we study the influence of defects in twisted bilayer graphene (TBG) using Raman spectroscopy. We explore the varied influence of defects on three characteristic Raman modes of both fully-defected TBG, with defects introduced in both layers, and half-defected TBG, with defects introduced in only a single layer. The resonance condition responsible for a strong enhancement of the $\mathrm{G}$ peak is sensitive to structural disorder and is quenched within a radius $\sim 3 \mathrm{~nm}$ of defects, while the twistangle dependence of the $2 \mathrm{D}$ peak is influenced only at the site of structural disorder $(\sim 1 \mathrm{~nm}$ radius).
\end{abstract}

\section{Introduction}

Building upon the development of graphene[1] and other two-dimensional (2D) materials,[2,3] the diversity of layered systems has expanded through the stacking of planar materials into van der Waals (vdW) films and heterosystems.[4,5] Among the most elementary of such systems is a stack of two rotated, electronically-coupled graphene sheets: twisted bilayer graphene (TBG).[6-11] Adding one degree of freedom, the angle of misorientation between layers (twist angle), TBG allows the tailoring of a range of electronic and optical properties[12-15] while maintaining the linear band structure of graphene near the Dirac point. $[6,16,17]$

Raman spectroscopy provides a standard, non-destructive, and data-rich means of characterizing graphenic ( $s p^{2}$ carbon) systems, revealing structure, strain, doping, defect density, and defect type.[18-22] In the case of TBG, interlayer interactions modulate graphene's electronic structure,[8-10] particularly at energies where the Dirac cones overlap, leading to the emergence *Corresponding author: Tel: 202 404-4516. E-mail: scott.schmucker@gmail.com 
of twist angle-dependent saddle point van Hove singularities in the TBG density of states.[23] As a consequence, the Raman signature of TBG varies with twist angle $(\theta)$, producing a strong enhancement in the $\mathrm{G}$ peak for a critical angle at which the excitation laser is resonant with the van Hove singularities and exhibiting a complex influence on the $2 \mathrm{D}$ (or G') peak.

The D peak, forbidden by symmetry, is a measure of disorder in the Raman spectrum of graphene, and is also influenced by $\theta$ in TBG. As may be anticipated, this influence is similar to that exhibited on the second order 2D peak. However, in the case of the D peak, analysis is complicated by the concurrent influence of defects on the interlayer interactions inherent to TBG. As defects are introduced in one or both layers of TBG, the atomic and electronic structures of each layer, and of the coupled system, are altered.[24] As a result, an improved understanding of the Raman spectra of defective TBG is necessary to facilitate the study of defective or chemically-modified TBG and to extend earlier studies of ion irradiated graphene[19,25] and carbon nanotubes.[26,27] Already, the chemical-modification of TBG has been explored and modeled.[28,29] DFT simulations have elucidated the influence of structural defects on the electronic structure of TBG films. In recent work, M. Kalbac et al. studied the influence of ion bombardment on the Raman spectra of TBG for uncoupled, non-resonant twist domains, and revealed an enhanced self-annealing process in the lower layer, but did not report on the relationship between twist angle, defects, and coupling in the system.[30]

In this work, we explore the complex relationship between twist angle, defect density, and interlayer coupling in TBG using Raman spectroscopy and optical microscopy, with ion irradiation as our means to controllably introduce defects. We compare TBG having defects introduced in both layers with TBG having defects introduced in only one of the two layers.

\section{Sample Preparation}

Monolayer graphene is grown by low-pressure chemical vapor deposition in $\mathrm{Cu}$ foil “enclosures."[31,32] TBG is produced on a $100 \mathrm{~nm} \mathrm{SiO} 2 / \mathrm{Si}$ substrate by two sequential transfers of monolayer graphene using a poly(methyl methacrylate) (PMMA) film and wet chemical processing, as described previously.[11] In some cases, PMMA residues may persist following wet chemical transfer of graphene, the removal of which has motivated extensive process development.[33] However, the stacking of graphene into bilayer films can lead to a so-called 
"self-cleaning" process for interlayer residues[34]. We have previously shown that more than $95 \%$ of stacked bilayer films can be in intimate contact that leads to electronic hybridization, despite the relatively "dirty" transfer process.[11] During this self-cleaning process, interlayer residues diffuse into isolated hydrocarbon islands[34] (typically a few hundred nanometers in diameter) that can compose $<5 \%$ of the total area.

The resulting sample has both TBG and monolayer regions. Interlayer coupling within the TBG region can be confirmed by optical microscopy, where a "stained glass window" effect characteristic of TBG[11] is visible when domains are in resonance with specific photons energies of the white light (Figure 1a). We have previously quantified the relationship between "colored" TBG domains observed under white light microscopy and the twist angle specific to an individual color.[11] For example, the three primary TBG domains distinguishable in optical microscopy are: "blue" $\left(\theta=11^{\circ} \pm 1^{\circ}\right)$, "red" $\left(\theta=13^{\circ} \pm 1^{\circ}\right)$, and "yellow" $\left(\theta=15^{\circ} \pm 1^{\circ}\right)$ (Figure 1a), where these twist angles have been independently confirmed via diffraction measurements.[11] We note that twist angle variations resulting from local wrinkling and morphological defects of the graphene films can introduce rotational disorder across a single domain[35]. As such, for the work presented here we select only those TBG domains that express a single uniform color in the optical microscope. In the remaining colorless domains $\left(\theta<10^{\circ}\right.$ and $\left.\theta>16^{\circ}\right)$, the resonance condition occurs at wavelengths (i.e., energies) outside of the visible spectrum.

To further identify the twist angle of colorless TBG domains, we use Raman spectroscopy. We perform measurements of TBG by confocal Raman spectroscopy using a custom system with $488 \mathrm{~nm}(2.54 \mathrm{eV})$ laser light focused on the sample with a $50 \times$ objective lens $(\mathrm{NA}=0.65)$ to a spot size of approximately $0.4 \mathrm{um}$ and power of approximately $3 \mathrm{~mW}$ at the sample. With Raman mapping, we collect micro-Raman spectra across multiple twist angle domains. By measuring the integrated intensity of the 2D peak (Int(2D)), together with optical microscopy, we can distinguish five twist angle ranges: "large" $\left(>16^{\circ}\right)$, "small" $\left(<10^{\circ}\right)$, and three distinctly colored domains with twist angles between $10^{\circ}$ and $16^{\circ}$ described above.[10]

A range of defect densities was selected to span the first two stages of amorphization in graphitic systems (i.e., 'Stage 1' and 'Stage 2').[36] In Stage 1, where defect densities are less than $\approx 1 \times 10^{13} \mathrm{~cm}^{-2}$, graphene becomes increasingly nanocrystalline due to the formation of spatially isolated defects. For higher defect densities, Stage 2, graphene becomes increasingly amorphous 
as defected regions begin to coalesce. This trend can be observed in the Raman spectra and is characterized by an initial increase in D/G intensity ratio in Stage 1 (up to about 3:1) followed by a decline and leveling off at a 1:1 ratio in Stage 2. There is also increased dispersion of the $G$ peak in Stage 2.[36] Unique to TBG, we note that as the defect density increases, the "stained glass window" optical effect described earlier is lost, leaving the TBG film colorless (Figure 1b).

\subsection{Fully-Defected Twisted Bilayer Graphene}

For fully-defected TBG (fTBG), defects are simultaneously introduced into both layers by 35 $\mathrm{keV} \mathrm{C}$ ion irradiation using a range of known fluences $\left(1 \times 10^{13} \mathrm{~cm}^{-2}\right.$ to $\left.1 \times 10^{15} \mathrm{~cm}^{-2}\right)$, producing fTBG with a range of defect densities $\left(6 \times 10^{11} \mathrm{~cm}^{-2}\right.$ to $6 \times 10^{13} \mathrm{~cm}^{-2}$ per layer $)$. Defect densities are estimated from the $\mathrm{D} / \mathrm{G}$ ratio of Raman spectra taken in neighboring monolayer regions, on the basis of earlier published results correlating Raman spectra with defect densities measured by scanning tunneling microscopy.[19] Our estimated defect densities correspond to a defect generation yield of $6 \%$, which is consistent with previous experimental results and theoretical predictions for equivalent ion bombardment conditions.[25,37]

Prior to ion irradiation, interlayer coupling in TBG is confirmed by Raman spectroscopy. We observe the characteristic resonant enhancement of the Raman G peak and twist angle dependence of the 2D peak, $[9,10]$ as will be discussed later. Additionally, we note the appearance of the twist angle-dependent $\mathrm{R}$ and $\mathrm{R}^{\prime}$ peaks.[38] The $\mathrm{R}$ peak is seen for twist angles near the resonance, where its intensity is enhanced, and $\mathrm{R}^{`}$ for some small twist angles as a shoulder on the $\mathrm{G}$ peak. By analyzing the position of the $\mathrm{R}$ peak and comparing with earlier studies,[39] we verify that the twist angle ranges for yellow- and red-colored domains agree with those measured by LEED in earlier work.[11] We also note the appearance of a peak near 1340 $\mathrm{cm}^{-1}$ which has been previously identified as a "D-like" mode activated not by structural defects but by the interlayer periodic potential of TBG.[39,40] As expected, this mode appears at large twist angles, but is not clearly observed at smaller twist angles (including all angles below $16^{\circ}$ ). These spectral features are demonstrated in Figure 1c, which includes representative spectra of pristine TBG for a collection of twist angles.

It is known that the intensity ratio of the D to D` peaks provides insight into the nature of defects present in monolayer graphene. For vacancy-like defects, this ratio is $\sim 7$, increasing for $\mathrm{sp}^{3}$-type 
defects and decreasing for grain boundaries.[21] After ion bombardment, Figure 1d presents a scatterplot of $D / G$ integrated intensity versus $D / G$ integrated intensity for TBG with a total defect density of $\sim 1 \times 10^{13} \mathrm{~cm}^{-2}$. D/D` ratios of 7 fall along the black line indicated on the plot, which tracks our data set closely for monolayer graphene, TBG with a large twist angle, and TBG at a resonant twist angle. This suggests that the defects present in our films are predominantly vacancy-type defects, as expected following bombardment with $35 \mathrm{keV} \mathrm{C}$ ions. Analysis of the D/D ratio for twist angles below resonance is complicated by the appearance of the $\mathrm{R}^{`}$ peak, which is convoluted with the $\mathrm{D}^{`}$ peak. As a result, for small twist angles the D/D` ratio is measured to be erroneously low $(\sim 5)$. This feature is believed to suggest only limitations in data analysis, rather than novel physics in the material system. A complete data set for all twist angle ranges is presented in Supplemental Figure 1.

\subsection{Half-Defected Twisted Bilayer Graphene}

We also consider half-defected TBG (hTBG), containing defects only in the lower graphene layer, which is produced by a slightly different processing order. To fabricate hTBG, CVD monolayer graphene is first transferred to $\mathrm{SiO}_{2}$ and defects are introduced by bombardment with a non-rastered $150 \mathrm{eV} \mathrm{Ar+} \mathrm{ion} \mathrm{beam} \mathrm{centered} \mathrm{on} \mathrm{the} \mathrm{sample.} \mathrm{The} \mathrm{Gaussian-like} \mathrm{beam} \mathrm{flux}$ results in spatially varying ion fluence over a millimeter length scale, leading to a range of defect densities $\left(2 \times 10^{12}\right.$ to $\left.9 \times 10^{13} \mathrm{~cm}^{-2}\right)$. Afterwards, a second pristine graphene layer is transferred on top of this defected layer, and local Raman mapping is performed in regions with the desired defect densities to identify large, small, and resonant TBG domains.

\section{Results and Discussion}

\subsection{Fully-Defected Twisted Bilayer Graphene}

To begin quantifying the effects of defects in TBG, we first explore the relationship between twist angle $(\theta)$ and the D peak and 2D peak full-width at half maximum (FWHM). Figure 2a shows both the D and 2D FWHM plotted in comparison to published 2D data (grey lines).[9] The similar trends between the 2D and D peaks are intuitive, owing to the fact that the 2D mode is the second order of the D mode. It is important to note, however, that the D peak arises only from Raman scattering in the activated region within a few nanometers of a defect site,[19] 
whereas the $2 \mathrm{D}$ peak is sensitive to scattering throughout the graphene lattice. Thus, if a lattice defect in one layer of TBG were to induce interlayer decoupling over a sufficiently large area, the D peak would be invariant with respect to $\theta$ (Supplemental Figure 2). In contrast, the observed twist angle dependence in our results suggests that the decoupling radius in fTBG is small relative to the radius of this activated region $(\sim 3 \mathrm{~nm})$ in which the D peak arises.

In Figure 2b, we follow the Raman spectral progression of TBG with large $\theta\left(>16^{\circ}\right)$ over a range of defect densities. These spectra correspond well to the characteristic Raman spectra of graphitic carbon evolving from highly ordered (Stage 1) to highly disordered (Stage 2).[36] Likewise, in Figure $2 \mathrm{c}$ we follow a similar progression for resonant $\theta\left(\sim 14^{\circ}\right)$, which has a substantial enhancement of the $\mathrm{G}$ peak in defect-free TBG. It should be noted that, while the Raman intensity scale is constant within each figure, the intensity scale is enlarged in Figure 2c to visualize the strong enhancement of the $\mathrm{G}$ peak (approximately $25 \times$ at resonance). A notable feature in this progression is the precipitous loss of resonant $G$ peak enhancement in fTBG, even at defect densities of $1 \times 10^{13}$ defects $/ \mathrm{cm}^{2}$. In Figure $2 \mathrm{~d}$, representative Raman spectra are shown for small $\theta\left(<10^{\circ}\right)$.

For increasing defect densities, Raman spectra evolve toward twist-angle independence as defected regions coalesce and the crystalline carbon film evolves towards amorphous carbon in Stage 2. In Figure 3a, we plot the relationship between $\theta$ and the $2 \mathrm{D}$ peak integrated intensity $(\operatorname{Int}(2 \mathrm{D}))$ with increasing defect density. Here, intensity is represented as a function of total defect density (defects $/ \mathrm{cm}^{2}$ ) contained in both layers (approximately twice that of the monolayer reference). Although the upper layer may possess a slightly higher defect density, resulting from enhanced self-annealing in the lower layer,[30] this asymmetry will not influence our subsequent analysis, which depends only upon the average defect density in the bilayer. For each defect density in Fig. 2a, Int(2D) is normalized relative to single-layer graphene (termed 'r.s.l'). The influence of defects on the $\theta$ dependence of $\operatorname{Int}(\mathrm{D})$ is presented in Figure $3 \mathrm{~b}$. In both cases, for nearly pristine films, $\operatorname{Int}(\mathrm{D})$ and $\operatorname{Int}(2 \mathrm{D})$ for $\theta<10^{\circ}$ are approximately equal to those of the monolayer, while for $\theta>16^{\circ} \operatorname{Int}(\mathrm{D})$ and $\operatorname{Int}(2 \mathrm{D})$ are approximately twice that of the monolayer.

As the film evolves from pristine graphene to amorphous carbon, $\operatorname{Int}(2 \mathrm{D})$ for $\theta>16^{\circ}$ falls to that of the monolayer, while for $\theta<10^{\circ}$ the relative intensity remains unchanged. The 
normalized G peak intensity (r.s.1.) on non-resonant twist domains exhibits a similar progression during ion bombardment (Supplemental Figure 3). The resonant angle depends on the laser wavelength and we observe no change in this angle as defect density increases.

Figure $3 c$ shows the evolution of the $G$ peak integrated intensity $(\operatorname{Int}(G))$ in a resonant domain as a function of the distance between defects $\left(\mathrm{L}_{\mathrm{D}}\right)$, including defects in both layers. As-fabricated TBG exhibits a strong enhancement of the $G$ peak at twist angles resonant with the excitation laser and the magnitude of this enhancement monotonically decreases with defect introduction throughout Stage 1. In pristine TBG, the $\operatorname{Int}(\mathrm{G})$ of a resonant domain $\left(\sim 14^{\circ}\right)$ is enhanced by a factor of approximately $30 \times$ relative to a non-resonant domain. At a defect density of $6 \times 10^{13} \mathrm{~cm}^{-}$ 2 (i.e., in Stage 2), $\operatorname{Int}(\mathrm{G})$ in the resonant domain has fallen to approximately 1 relative to a nonresonant domain. While some variation in $\operatorname{Int}(\mathrm{G})$ is expected within a single domain due to rotational disorder[35], the significant decrease in $\operatorname{Int}(\mathrm{G})$ here is attributed to introduced point defects.

In Figure 3d we show the influence of defect density on the TBG 2D and D peaks by plotting the ratio of integrated intensities for large $\left(\theta>16^{\circ}\right)$ and small $\left(\theta<10^{\circ}\right)$ twist angle domains (the coupling factor). This yields a self-consistent standard to quantify defect density in TBG, independent of a monolayer reference, and may be more representative under certain experimental conditions. For example, under chemical modification, monolayer and bilayer graphene exhibit different reactivity,[28,41,42] such that one cannot assume a comparable defect density on the monolayer, thus necessitating a TBG-based metric. For pristine TBG films, the coupling factor is $\sim 2.4$, and falls to $\sim 1$ in highly defected samples. The coupling factor decays with increasing defect density for both the $\mathrm{D}$ and $2 \mathrm{D}$ peaks. The only deviation from this curve occurs for the D peak at low defect density $\left(\sim 1 \times 10^{-12} \mathrm{~cm}^{-2}\right)$. The $\mathrm{D}$ coupling factor is not shown for pristine TBG, where this peak is negligible.

To better interpret these results, we model the system to consider the percentage of the TBG sheet decoupled by ion bombardment. We assume that each defect site induces decoupling over a circular area with some decoupling radius (r), where "decoupling" is defined by the evolution of the coupling factor towards 1 (i.e. Raman spectra become $\theta$ independent), and can therefore occur over different length scales depending on the spectral feature of interest. As G peak enhancement and 2D peak coupling factor exhibit distinct decoupling behavior, we assign each 
an independent decoupling radius, $\mathrm{r}_{\mathrm{G}}$ and $\mathrm{r}_{2 \mathrm{D}}$. After the introduction of $\sigma$ defects $/ \mathrm{cm}^{2}$, each of which induces decoupling within a radius $r$ from the defect site, the probability that a randomly selected location remains coupled (i.e. sits further than one decoupling radius from the nearest defect site) is:

$$
P_{\text {coupled }}=1-f_{\text {area }}=e^{-\pi \sigma r^{2}}
$$

Here, $\mathrm{f}_{\text {area }}$ is the fraction of the surface within one decoupling radius of the nearest defect. This model follows the work of Lucchese et al., where the area fraction of the structurally disordered region derives from an equivalent calculation.[19] Fitting this curve to our experimental data, we calculate the decoupling radius based on measurements of the $\mathrm{G}$ peak and, independently, the 2D peak. This does assume, however, that the defect sites in the upper and lower layers are uncorrelated. As it is possible for the generation of a defect in the upper layer to influence the creation of a defect in the lower layer (e.g. by collision cascades), we also consider the influence of this correlation on our calculation of decoupling radii. In the limit of full-correlation, where each defect in the lower layer resides directly below a defect in the upper layer, the effective total defect density (for purposes of decoupling) would be half of that calculated in our analysis. Although we do not measure the degree of correlation experimentally, by fitting Equation 1 to these fully-correlated plots (Supplemental Figure 4) we determine a range of decoupling radii which represent uncorrelated and correlated defects. We calculate a decoupling radius for the 2D peak ( $\mathrm{r}_{2 \mathrm{D}}$ ) between $0.82 \mathrm{~nm}$ and $1.2 \mathrm{~nm}$ (uncorrelated to perfectly correlated), and a decoupling radius for the $G$ peak $\left(r_{G}\right)$ between $2.2 \mathrm{~nm}$ and $3.1 \mathrm{~nm}$. These lengths are comparable to $r_{S}$ and $r_{D}$ reported by Lucchese et al. in a study of the D peak in monolayer graphene.[19] In that work, a single defect created a structurally disordered region $1 \mathrm{~nm}$ in radius, surrounded by an activated region $3 \mathrm{~nm}$ in radius which followed from the Raman relaxation length in their system. Our results suggest that the TBG 2D peak is modulated only by the introduction of structural disorder in the lattice of one or both layers, and that the layers of fTBG are not decoupled until the band structure of one or both layers is structurally modified. In contrast, the larger decoupling radius for the enhancement of the $\mathrm{G}$ peak implies that resonant enhancement near the Van Hove singularities is quenched in the symmetry-breaking activated region where the lifetime of the excited state is sufficiently long to allow interaction with the defect. As a result, the $\mathrm{G}$ peak resonant enhancement is more sensitive to changes in the atomic structure of 
one or both layers. The effects of resonance and decoupling can also be presented by the more conventional D/G intensity ratio, where $G$ peak enhancement exhibits a strong influence in resonant domains. The $\mathrm{D} / \mathrm{G}$ ratio for several representative twist angles is shown as a function of defect density in Supplemental Figure 5.

\subsection{Half-Defected Twisted Bilayer Graphene}

In contrast to the fTBG results, we find hTBG decouples at lower defect densities. Raman analysis of monolayer regions in both fTBG and hTBG provides a comparison between samples to ensure comparable defect densities (Figures $4 \mathrm{a}$ and $4 \mathrm{~b}$ ). Following the analysis employed for fTBG, we plot G peak enhancement factor (Figure 4c) and 2D peak coupling factor (Figure 4d) for hTBG as a function of defect density. The most obvious difference for hTBG is the rapid onset of decoupling. For films with $1 \times 10^{13}$ defects $/ \mathrm{cm}^{2}$ the Raman spectra have nearly achieved full twist angle independence, a state not achieved in fTBG until introducing a factor $10 \times$ more defects $\left(1 \times 10^{14}\right.$ defects $\left./ \mathrm{cm}^{2}\right)$. Fitting with Equation 1, we observe large decoupling lengths of $3.4 \mathrm{~nm}$ for both the $\mathrm{G}$ and 2D peaks (fits shown). Unlike fTBG, we observe quantitatively similar behavior for $\mathrm{G}$ and $2 \mathrm{D}$ peaks in $\mathrm{hTBG}$, suggesting that a different mechanism contributes to decoupling in this system. The D/G intensity ratios for hTBG are shown in Supplemental Figure 6. Unlike fTBG, and due to the rapid onset of decoupling, the three twist angle ranges shown exhibit a similar trend in the $\mathrm{D} / \mathrm{G}$ intensity ratio. Decoupling radii calculated in this work for both fTBG and hTBG are summarized in Table 1.

We cannot rule out the possibility that the different fabrication procedures for hTBG and fTBG could lead to slightly different coupling behaviors. Under certain conditions, water[43,44] and hydrocarbons [34] are encapsulated below and between graphene sheets. In fTBG samples, formed by stacking pristine monolayers, the resulting bilayer has $>95 \%$ of the film directly coupled, with localized islands of debris formed through an apparent "self-cleaning" process.[34] In contrast, hTBG is formed by first introducing defects in the bottom monolayer and then transferring a pristine layer on top. The defects in the bottom layer may influence the diffusion of interlayer adsorbates into localized islands (i.e. the so called "self-cleaning" process). If the defects in the bottom layer of hTBG can trap a small volume of material averaging $3.4 \mathrm{~nm}$ in radius surrounding each defect site, then decoupling of the bilayer could occur by physical separation from interlayer material. Since the trapping of intercalated material by this process 
simply reduces the total area of coupled TBG, if the separation area is larger than the activated region surrounding the defect, both the $\mathrm{G}$ and 2D Raman peaks will be influenced identically. Furthermore, we note that the D peak coupling factor is lower in hTBG than fTBG, suggesting increased prevalence of the decoupling mechanism shown in Supplemental Figure 2a, and consistent with the trapping of interlayer material.

\section{Conclusions}

In summary, we have measured the influence of structural defects on the Raman spectra of TBG, and the interlayer decoupling induced by ion bombardment. The differing influence of disorder on the twist angle dependence of graphene Raman modes is demonstrated and explained. Defect introduction by ion bombardment induces interlayer decoupling on the scale of $1 \mathrm{~nm}$, while dampening of the characteristic $G$ peak resonance extends into the activated region within $\sim 3 \mathrm{~nm}$ of a vacancy. We find that the influence of defects pre-existing before layer stacking is greater, possibly due to the trapping of interlayer material at defect sites. The results of this study provide a framework for the characterization of defected TBG, a foundation for the more general study of defects in coupled van der Waals heterostructures, and a material system with potential applications in a wide range of fields, including sensing and optoelectronics.

\section{Acknowledgements}

The work at the Naval Research Laboratory was supported by the Office of Naval Research, the Defense Threat Reduction Agency, and the NRL Nanoscience Institute. This research was performed while S.W. Schmucker held a National Research Council Associateship Award at the Naval Research Laboratory. 


\section{References}

[1] Novoselov KS, Geim AK, Morozov SV, Jiang D, Zhang Y, Dubonos SV, et al. Electric Field Effect in Atomically Thin Carbon Films. Science 2004;306:666-9. doi:10.1126/science.1102896.

[2] Neto AHC, Novoselov K. New directions in science and technology: two-dimensional crystals. Rep Prog Phys 2011;74:082501. doi:10.1088/0034-4885/74/8/082501.

[3] Butler SZ, Hollen SM, Cao L, Cui Y, Gupta JA, Gutiérrez HR, et al. Progress, Challenges, and Opportunities in Two-Dimensional Materials Beyond Graphene. ACS Nano 2013;7:2898-926. doi:10.1021/nn400280c.

[4] Geim AK, Grigorieva IV. Van der Waals heterostructures. Nature 2013;499:419-25. doi:10.1038/nature12385.

[5] Gao G, Gao W, Cannuccia E, Taha-Tijerina J, Balicas L, Mathkar A, et al. Artificially Stacked Atomic Layers: Toward New van der Waals Solids. Nano Lett 2012;12:3518-25. doi:10.1021/nl301061b.

[6] Lopes dos Santos JMB, Peres NMR, Castro Neto AH. Graphene Bilayer with a Twist: Electronic Structure. Phys Rev Lett 2007;99:256802. doi:10.1103/PhysRevLett.99.256802.

[7] Schmidt H, Lüdtke T, Barthold P, McCann E, Fal'ko VI, Haug RJ. Tunable graphene system with two decoupled monolayers. Appl Phys Lett 2008;93:172108. doi:10.1063/1.3012369.

[8] Havener RW, Zhuang H, Brown L, Hennig RG, Park J. Angle-Resolved Raman Imaging of Interlayer Rotations and Interactions in Twisted Bilayer Graphene. Nano Lett 2012;12:3162-7. doi:10.1021/n1301137k.

[9] Kim K, Coh S, Tan LZ, Regan W, Yuk JM, Chatterjee E, et al. Raman Spectroscopy Study of Rotated Double-Layer Graphene: Misorientation-Angle Dependence of Electronic Structure. Phys Rev Lett 2012;108:246103. doi:10.1103/PhysRevLett.108.246103.

[10] Coh S, Tan LZ, Louie SG, Cohen ML. Theory of the Raman spectrum of rotated doublelayer graphene. Phys Rev B 2013;88:165431. doi:10.1103/PhysRevB.88.165431.

[11] Robinson JT, Schmucker SW, Diaconescu CB, Long JP, Culbertson JC, Ohta T, et al. Electronic Hybridization of Large-Area Stacked Graphene Films. ACS Nano 2013;7:63744. doi:10.1021/nn304834p.

[12] Tabert CJ, Nicol EJ. Optical conductivity of twisted bilayer graphene. Phys Rev B 2013;87:121402. doi:10.1103/PhysRevB.87.121402.

[13] Moon P, Koshino M. Optical absorption in twisted bilayer graphene. Phys Rev B 2013;87:205404. doi:10.1103/PhysRevB.87.205404.

[14] Havener RW, Liang Y, Brown L, Yang L, Park J. Van Hove Singularities and Excitonic Effects in the Optical Conductivity of Twisted Bilayer Graphene. Nano Lett 2014;14:33537. doi:10.1021/nl500823k.

[15] Wang Y, Ni Z, Liu L, Liu Y, Cong C, Yu T, et al. Stacking-Dependent Optical Conductivity of Bilayer Graphene. ACS Nano 2010;4:4074-80. doi:10.1021/nn1004974.

[16] Hass J, Varchon F, Millán-Otoya JE, Sprinkle M, Sharma N, de Heer WA, et al. Why Multilayer Graphene on $4 \mathrm{H}-\mathrm{SiC}\left(0001^{-}\right)$Behaves Like a Single Sheet of Graphene. Phys Rev Lett 2008;100:125504. doi:10.1103/PhysRevLett.100.125504.

[17] Shallcross S, Sharma S, Pankratov OA. Quantum Interference at the Twist Boundary in Graphene. Phys Rev Lett 2008;101:056803. doi:10.1103/PhysRevLett.101.056803. 
[18] Ferrari AC, Basko DM. Raman spectroscopy as a versatile tool for studying the properties of graphene. Nat Nanotechnol 2013;8:235-46. doi:10.1038/nnano.2013.46.

[19] Lucchese MM, Stavale F, Ferreira EHM, Vilani C, Moutinho MVO, Capaz RB, et al. Quantifying ion-induced defects and Raman relaxation length in graphene. Carbon 2010;48:1592-7. doi:10.1016/j.carbon.2009.12.057.

[20] Cançado LG, Jorio A, Ferreira EHM, Stavale F, Achete CA, Capaz RB, et al. Quantifying Defects in Graphene via Raman Spectroscopy at Different Excitation Energies. Nano Lett 2011;11:3190-6. doi:10.1021/nl201432g.

[21] Eckmann A, Felten A, Mishchenko A, Britnell L, Krupke R, Novoselov KS, et al. Probing the Nature of Defects in Graphene by Raman Spectroscopy. Nano Lett 2012;12:3925-30. doi:10.1021/nl300901a.

[22] Lee JE, Ahn G, Shim J, Lee YS, Ryu S. Optical separation of mechanical strain from charge doping in graphene. Nat Commun 2012;3:1024. doi:10.1038/ncomms2022.

[23] Li G, Luican A, Lopes dos Santos JMB, Castro Neto AH, Reina A, Kong J, et al. Observation of Van Hove singularities in twisted graphene layers. Nat Phys 2010;6:109-13. doi:10.1038/nphys 1463.

[24] Ulman K, Narasimhan S. Point defects in twisted bilayer graphene: A density functional theory study. Phys Rev B 2014;89:245429. doi:10.1103/PhysRevB.89.245429.

[25] Buchowicz G, Stone PR, Robinson JT, Cress CD, Beeman JW, Dubon OD. Correlation between structure and electrical transport in ion-irradiated graphene grown on $\mathrm{Cu}$ foils. Appl Phys Lett 2011;98:032102. doi:10.1063/1.3536529.

[26] Rossi JE, Cress CD, Helenic AR, Schauerman CM, DiLeo RA, Cox ND, et al. Ion irradiation of electronic-type-separated single wall carbon nanotubes: A model for radiation effects in nanostructured carbon. J Appl Phys 2012;112:034314. doi:10.1063/1.4739713.

[27] Rossi JE, Cress CD, Merrill A, Soule KJ, Cox ND, Landi BJ. Intrinsic diameter dependent degradation of single-wall carbon nanotubes from ion irradiation. Carbon 2015;81:488-96. doi:10.1016/j.carbon.2014.09.081.

[28] Ek Weis J, Costa SD, Frank O, Bastl Z, Kalbac M. Fluorination of Isotopically Labeled Turbostratic and Bernal Stacked Bilayer Graphene. Chem - Eur J 2014:n/a - n/a. doi:10.1002/chem.201404813.

[29] Muniz AR, Maroudas D. Superlattices of Fluorinated Interlayer-Bonded Domains in Twisted Bilayer Graphene. J Phys Chem C 2013;117:7315-25. doi:10.1021/jp310184c.

[30] Kalbac M, Lehtinen O, Krasheninnikov AV, Keinonen J. Ion-Irradiation-Induced Defects in Isotopically-Labeled Two Layered Graphene: Enhanced In-Situ Annealing of the Damage. Adv Mater 2013;25:1004-9. doi:10.1002/adma.201203807.

[31] Li X, Cai W, An J, Kim S, Nah J, Yang D, et al. Large-Area Synthesis of High-Quality and Uniform Graphene Films on Copper Foils. Science 2009;324:1312-4. doi:10.1126/science.1171245.

[32] Li X, Magnuson CW, Venugopal A, Tromp RM, Hannon JB, Vogel EM, et al. Large-Area Graphene Single Crystals Grown by Low-Pressure Chemical Vapor Deposition of Methane on Copper. J Am Chem Soc 2011;133:2816-9. doi:10.1021/ja109793s.

[33] Lin Y-C, Lu C-C, Yeh C-H, Jin C, Suenaga K, Chiu P-W. Graphene Annealing: How Clean Can It Be? Nano Lett 2012;12:414-9. doi:10.1021/nl203733r.

[34] Haigh SJ, Gholinia A, Jalil R, Romani S, Britnell L, Elias DC, et al. Cross-sectional imaging of individual layers and buried interfaces of graphene-based heterostructures and superlattices. Nat Mater 2012;11:764-7. doi:10.1038/nmat3386. 
[35] Beechem TE, Ohta T, Diaconescu B, Robinson JT. Rotational Disorder in Twisted Bilayer Graphene. ACS Nano 2014;8:1655-63. doi:10.1021/nn405999z.

[36] Lehtinen O, Kotakoski J, Krasheninnikov AV, Tolvanen A, Nordlund K, Keinonen J. Effects of ion bombardment on a two-dimensional target: Atomistic simulations of graphene irradiation. Phys Rev B 2010;81:153401. doi:10.1103/PhysRevB.81.153401.

[37] Carozo V, Almeida CM, Ferreira EHM, Cançado LG, Achete CA, Jorio A. Raman Signature of Graphene Superlattices. Nano Lett 2011;11:4527-34. doi:10.1021/nl201370m.

[38] Carozo V, Almeida CM, Fragneaud B, Bedê PM, Moutinho MVO, Ribeiro-Soares J, et al. Resonance effects on the Raman spectra of graphene superlattices. Phys Rev B 2013;88:085401. doi:10.1103/PhysRevB.88.085401.

[39] Gupta AK, Tang Y, Crespi VH, Eklund PC. Nondispersive Raman \$D \$ band activated by well-ordered interlayer interactions in rotationally stacked bilayer graphene. Phys Rev B 2010;82:241406. doi:10.1103/PhysRevB.82.241406.

[40] Ferrari AC, Robertson J. Interpretation of Raman spectra of disordered and amorphous carbon. Phys Rev B 2000;61:14095-107. doi:10.1103/PhysRevB.61.14095.

[41] Ryu S, Han MY, Maultzsch J, Heinz TF, Kim P, Steigerwald ML, et al. Reversible Basal Plane Hydrogenation of Graphene. Nano Lett 2008;8:4597-602. doi:10.1021/nl802940s.

[42] Diankov G, Neumann M, Goldhaber-Gordon D. Extreme Monolayer-Selectivity of Hydrogen-Plasma Reactions with Graphene. ACS Nano 2013;7:1324-32. doi:10.1021/nn304903m.

[43] Xu K, Cao P, Heath JR. Graphene Visualizes the First Water Adlayers on Mica at Ambient Conditions. Science 2010;329:1188-91. doi:10.1126/science.1192907.

[44] He KT, Wood JD, Doidge GP, Pop E, Lyding JW. Scanning Tunneling Microscopy Study and Nanomanipulation of Graphene-Coated Water on Mica. Nano Lett 2012;12:2665-72. doi:10.1021/nl202613t. 

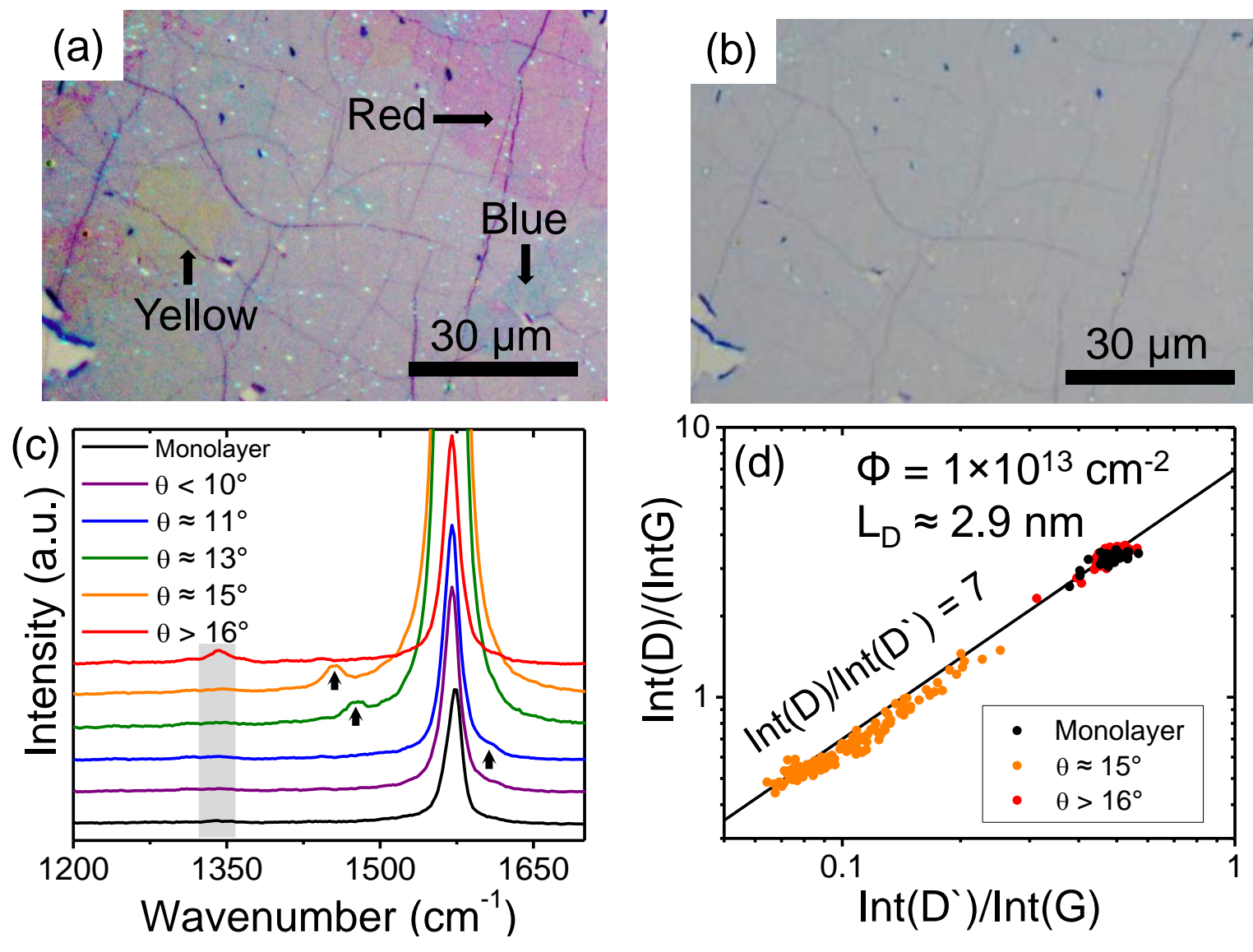

Figure 1: Optical microscopy and Raman spectroscopy of twisted bilayer graphene. a, Unfiltered optical image of TBG on 100nm SiO2/Si before ion bombardment showing the "yellow", "red", and "blue" domains observed under white light microscopy . b, Equivalent optical image of the same area in (a) following ion bombardment with a fluence of $1 \times 10^{15}$ $\mathrm{cm}^{-2}$ (estimated defect density of $6 \times 10^{13} \mathrm{~cm}^{-2}$ per layer). c) Representative spectra for several twist angles in pristine TBG. $\mathrm{R}\left(\omega<\omega_{\mathrm{G}}\right)$ and $\mathrm{R}^{\prime}\left(\omega>\omega_{\mathrm{G}}\right)$ peaks are indicated by black arrows, and the spectral region occupied by the D-like mode specific to TBG is indicated by a grey bar. d) The relative integrated intensities of the D and G modes and of the D`and G modes respectively for fTBG following ion bombardment to a fluence of $1 \times 10^{13} \mathrm{~cm}^{-2}$ (corresponding to a defect spacing of $2.9 \mathrm{~nm}$, including both layers). A solid black line is shown which corresponds to a D/D` ratio of 7 and follows the data closely. The resonance condition arising near $14^{\circ}$ (orange points) reduces the $\mathrm{D} / \mathrm{G}$ and $\mathrm{D} / \mathrm{G}$ ratios but does not substantially influence the $\mathrm{D} / \mathrm{D}^{`}$ ratio. 

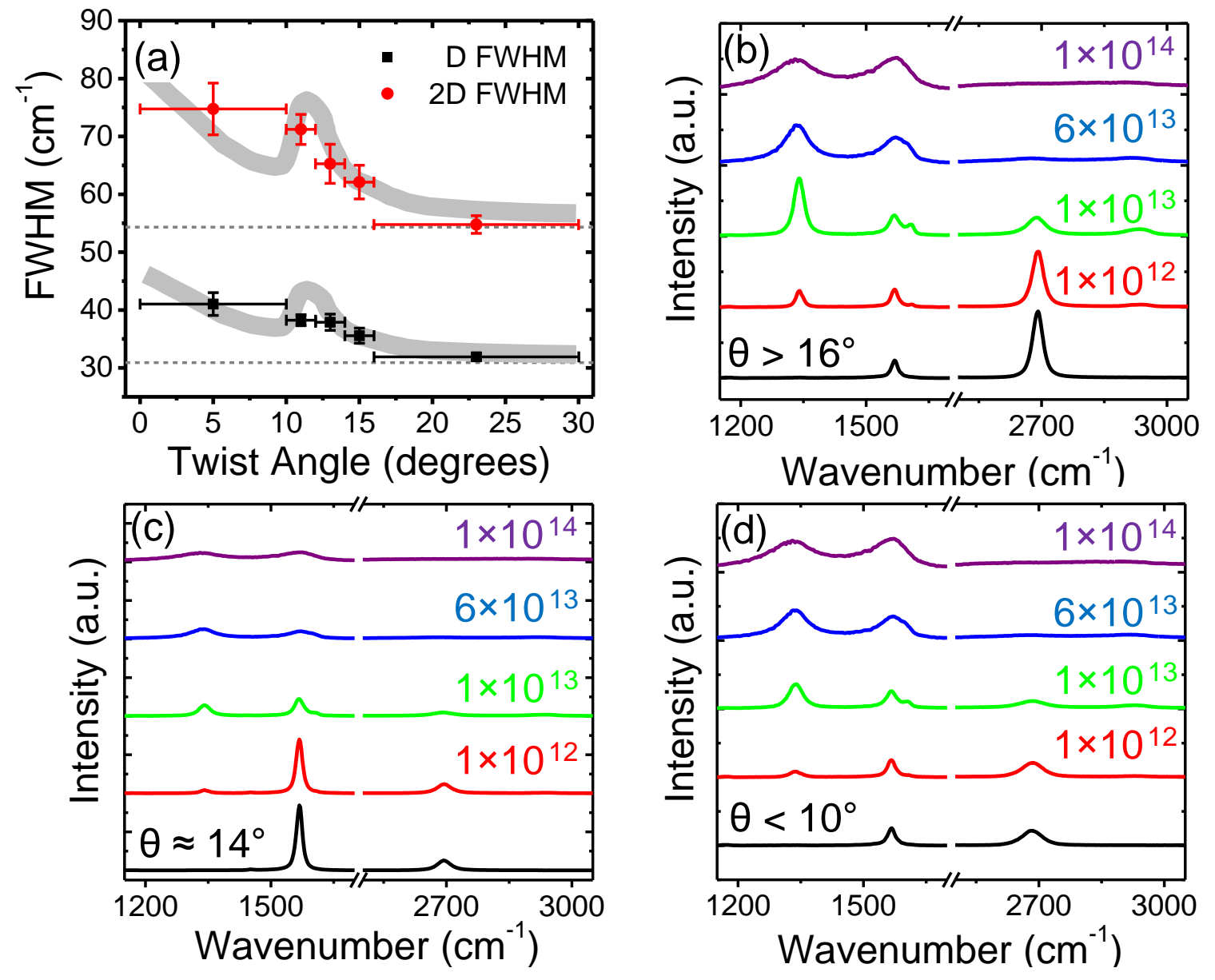

Figure 2: Raman spectra of fTBG as a function of twist angle. Defect densities indicated on each curve are total defect densities, including both graphene layers in units of $\mathrm{cm}^{-2}$. The defect density is approximately twice that of the graphene monolayer. a, Variation in D and 2D peak width as a function of twist angle. Samples were previously ion bombarded with a fluence of $1 \times 10^{14}$ (estimated defect density of $6 \times 10^{12}$ ). Data are shown together with data from reference [9] (solid grey lines) which has been scaled to bring the curves in registry with the FWHM from our monolayer reference (indicated by grey dotted lines). b, Representative Raman spectra of TBG with a large misorientation angle $\left(>16^{\circ}\right)$ for a range of defect densities. c, Representative spectra at the critical twist angle, resonant with $488 \mathrm{~nm}$ laser excitation (near $14^{\circ}$ ). d, Representative spectra with a small misorientation angle $\left(<10^{\circ}\right)$. 

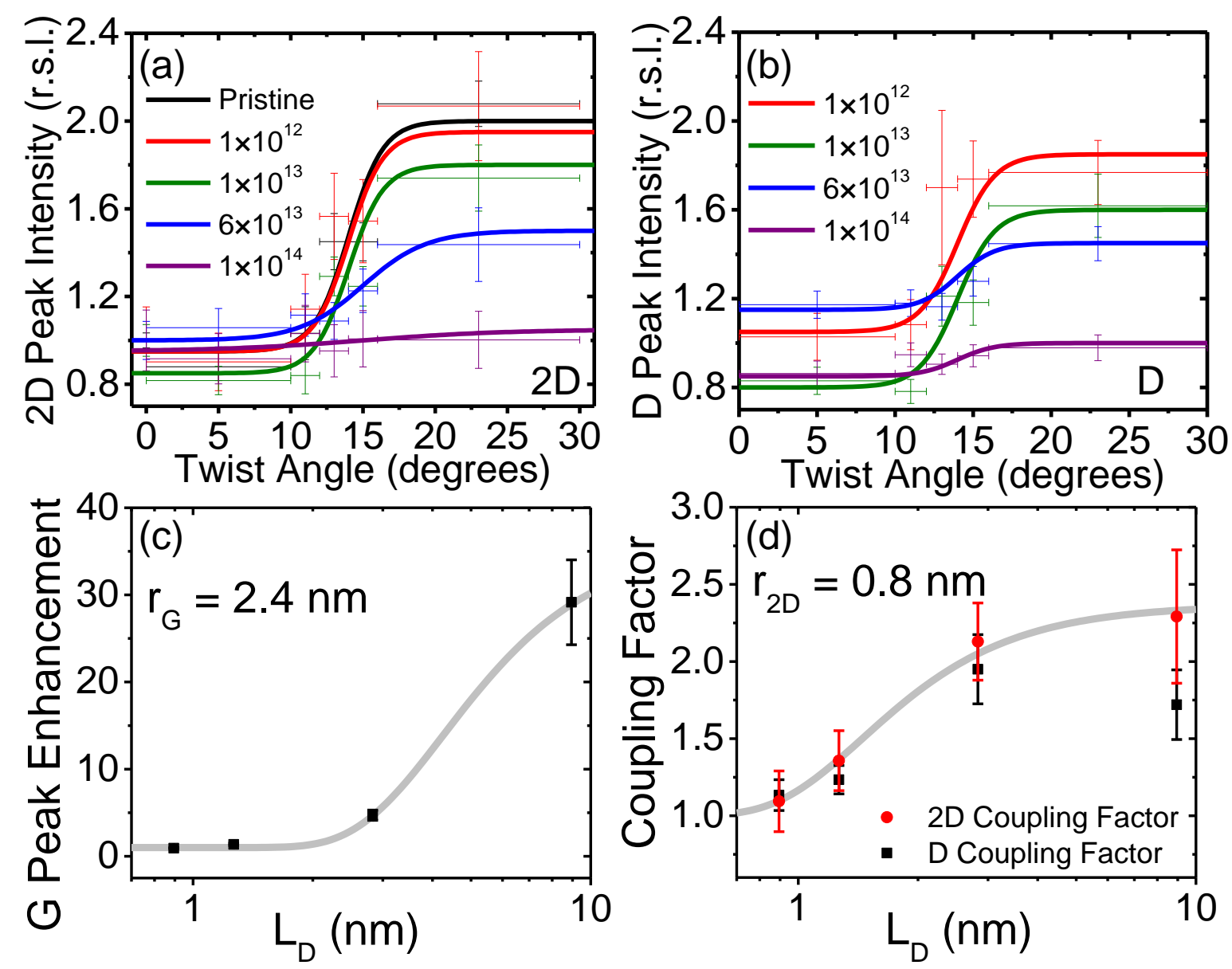

Figure 3: Variation in the Raman spectra of fTBG as a function of defect density. a, Variations in 2D peak intensity as a function of twist angle and defect density. For each defect density, average 2D intensity is shown for five distinct twist angle ranges. Solid lines are included as a guide to the eye. Defect densities indicated are total defect densities, including both graphene layers. b, Variation in D peak intensity are similarly represented. Again, solid lines are included as a guide to the eye. $\mathrm{D}$ peak intensity data is not included for pristine graphene. c, Variations in $\mathrm{G}$ peak enhancement as a function of distance between defects (including both graphene layers). Data are fitted based on Equation $1\left(P_{\text {coupled }} \times G_{\text {Max }}+\left(1-P_{\text {coupled }}\right) \times G_{\text {min }}\right) u$ using a radius of $2.2 \mathrm{~nm}$ (grey curve). d, Variation in D and 2D peak coupling factor as a function of distance between defects (including both graphene layers). 2D peak intensity is fitted based on Equation 1 with a radius of $0.82 \mathrm{~nm}$ (grey curve). No fit is presented for the D peak, but for defect densities above $\sim 1 \times 10^{13} \mathrm{~cm}^{-2}$, the $\mathrm{D}$ peak coupling factor follows that of the $2 \mathrm{D}$ peak. 

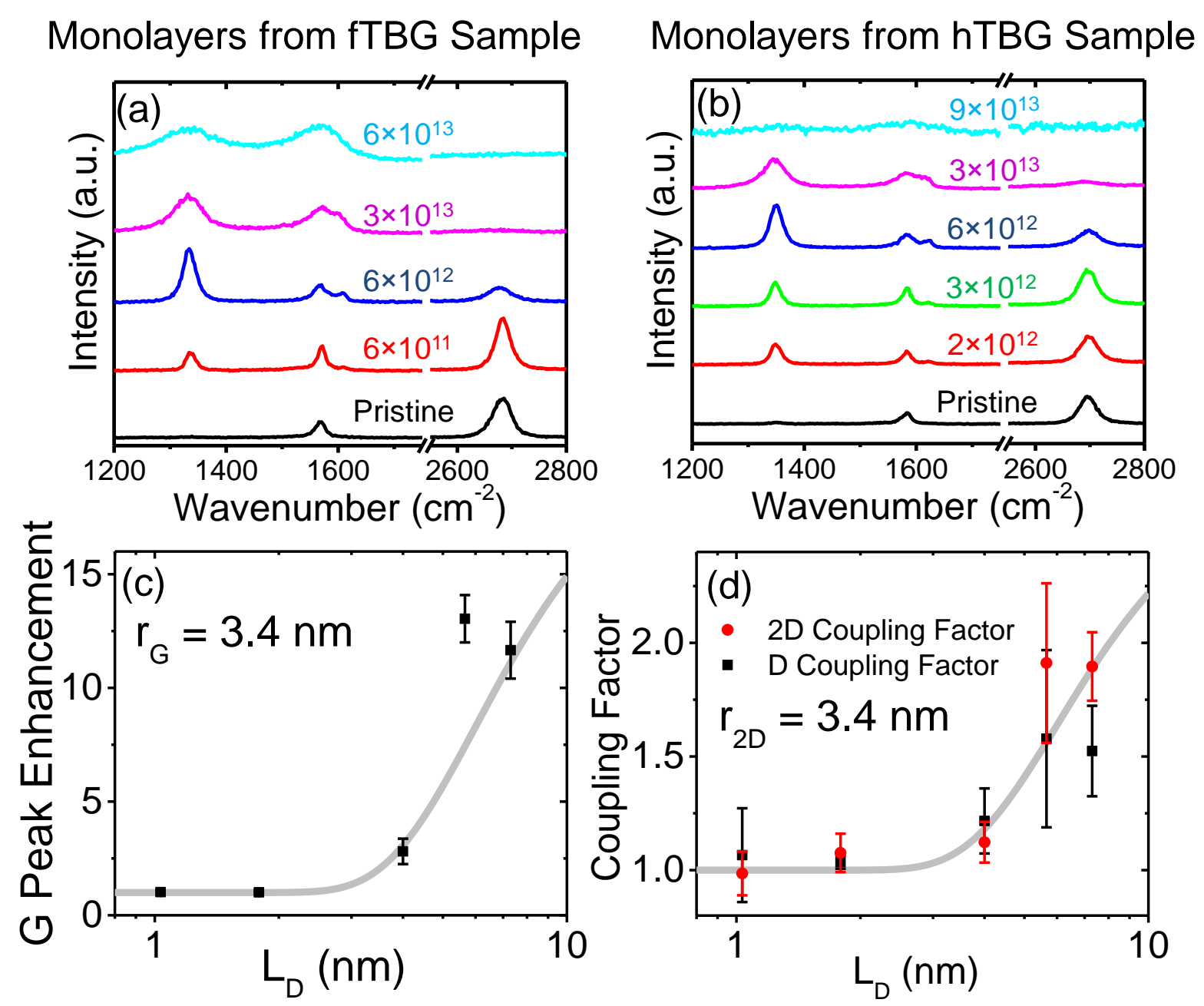

Figure 4: Raman spectra of hTBG as a function of defect density. Defect densities indicated on each curve are in units of $\mathrm{cm}^{-2}$. a, Representative Raman spectra of the monolayer reference samples from our fTBG sample, from which defect density is estimated. b, Representative Raman spectra of our hTBG sample before transfer of the pristine upper layer, by which defect densities are estimated in agreement with our fTBG sample. c, Variation in G peak enhancement in hTBG as a function of defect density, with the grey curve fit calculated based on Equation 1 for a radius of $3.5 \mathrm{~nm}$. d, Variation in D and 2D coupling factors for hTBG, with the grey curve fit based on Equation 1, also for a radius of $3.5 \mathrm{~nm}$. 
Table 1: Fitting parameters for decoupling of defected TBG.

\begin{tabular}{|c|c|c|}
\hline Sample & Parameter & Length \\
\hline $\begin{array}{c}\text { Fully-Defected } \\
\text { Uncorrelated Defects }\end{array}$ & $\mathrm{r}_{2 \mathrm{D}}$ & $0.82 \mathrm{~nm}$ \\
\cline { 2 - 3 } & $\mathrm{r}_{\mathrm{G}}$ & $2.4 \mathrm{~nm}$ \\
\hline $\begin{array}{c}\text { Fully-Defected } \\
\text { Correlated Defects }\end{array}$ & $\mathrm{r}_{2 \mathrm{D}}$ & $1.2 \mathrm{~nm}$ \\
\cline { 2 - 3 } & $\mathrm{r}_{\mathrm{G}}$ & $3.4 \mathrm{~nm}$ \\
\hline Half-Defected & $\mathrm{r}_{2 \mathrm{D}}$ & $3.4 \mathrm{~nm}$ \\
\cline { 2 - 3 } & $\mathrm{r}_{\mathrm{G}}$ & $3.4 \mathrm{~nm}$ \\
\hline
\end{tabular}

